\title{
Migration ist in dieser Stadt eine Tatsache

\author{
Urban politics of citizenship in der neoliberalen Stadt
}

\author{
Mathias Rodatz
}

Der Beitrag rekonstruiert die politics of citizenship aktueller städtischer Integrationsprogrammatiken. Im Zuge der Anpassung des Integrationsparadigmas an die Logik der , unternehmerischen Stadt' werden nationale Traditionen von Stadtpolitik infrage gestellt. Entsprechende Konzepte problematisieren (Post-)Migrant_innen als zu aktivierende Stadtbürger_innen. Anstatt diese Beobachtung zu einer negativen Kritik an Neoliberalisierungsprozessen kurzzuschließen, schlägt der Beitrag vor, die neuen staatlichen Problematisierungen als (möglicherweise nützliche) Bedingungen für Kämpfe um eine gleichberechtigte Teilhabe in der Stadt zu verstehen. Anhand der Genese des Frankfurter Konzepts wird gezeigt, dass unter neoliberalen Umständen postnationale wissenschaftliche Konzepte für die Regierung der Stadt mobilisiert werden. Damit repräsentiert das Konzept (Post-)Migration als Bestandteil einer ,vielfältigen' Normalität - an der sich Stadtpolitik in Zukunft auch politisch messen lassen müsste.

Ersteinreichung: 28. Mai 2014; Veröffentlichung online: 23. Dezember 2014

An english abstract can be found at the end of the document.

Diagnosen wie jene von Frankfurts Oberbürgermeister Wallmann, der die (Post-)Migrant_innen[1] der Stadt noch in den 1980er Jahren als „soziale Risiken in einem nie dagewesenen Ausmaß“ und „politischen und sozialen Sprengstoff“ (zit. n. Schipper 2013: 159) repräsentieren konnte, trieben lange das deutsche Mantra vom ,Nichteinwanderungsland' an. Sein halbherziges politisches Ende spiegelt sich bis heute regelmäßig in „urbanen Paniken“, die im Zeichen eines kulturalistischen Integrationsparadigmas Teile des transnationalen Alltags deutscher Städte als „Parallelgesellschaften“ skandalisieren (Tsianos 2013, Ronneberger/Tsianos 2009). Die heutige Integrationsdezernentin der Stadt Frankfurt am Main wurde kurz vor der Wahl in ihre Funktion mit einem Ausspruch bekannt, der ein deutlich anderes städtisches Selbstverständnis offenbart: „Migration in Frankfurt ist eine Tatsache. Wenn Ihnen das nicht gefällt, müssen sie woanders hinziehen“ (Eskandari-Grünberg zit. n. Topçu 2007). Über das von der Dezernentin verantwortete Integrations- und Vielfaltskonzept der Stadt urteilt Necla Kelek als eine prominente Vertreterin des nationalen Integrationsparadigmas[2] dann auch in bekannt, panischer" Manier: „Wer Vielfalt als Tatsache setzt und daraus keine Gemeinschaft anstrebt, deutet schlicht das Staatsziel um“ (2011). Das Konzept sei eine Gefahr für die „Integration“, weil die Stadt hier das staatliche Ziel einer nach „nationaler Identität“ vereinten Gesellschaft aufgebe: 
„Die Verfasser formulieren kein Integrationskonzept, sondern ein Programm, das den Migranten ein Leben in Frankfurt nach ihren eigenen Vorstellungen ermöglichen soll. [...] In diesem Konzept ist Integration eine Bringschuld [des Staates], und dabei wird weder eine nationale Identität noch die Teilhabe an Sprache und Kultur gefordert. Dieses Konzept atmet das Ideal des Internationalismus, das alle Völker und Nationen globalisiert; Frankfurt als Global City.“ (Ebd.)

Dass Kelek das Frankfurter Integrationskonzept als Gegenstand ihrer Polemik[3] aus Perspektive des nationalen Integrationsparadigmas wählt, lässt aufhorchen. Diese Kritik wird im Folgenden zum Anlass genommen, sich die Entwicklung städtischer Integrationspolitiken der vergangenen Jahre mit Blick auf Frankfurt noch einmal genauer anzuschauen.

Feststeht, dass kommunale Integrationspolitiken in den letzten 20 Jahren eine Aufwertung erfahren haben. Ein Teil der angewandten Forschung sieht in dieser Entwicklung einen „Paradigmenwechsel“ - aus einer fragmentierten Landschaft unterschiedlicher lokalpolitischer Traditionen habe sich ein dezidiertes Politikfeld entwickelt, das mit einem Perspektivwechsel verbunden sei, der nicht nur Integration als zentrale kommunale Aufgabe begreife, sondern auch deren traditionelle ,Defizitorientierung' aufgebe (Häußermann/ Kapphan 2008). Andere Perspektiven sehen im ,Paradigmenwechsel' lediglich eine semantische Volte, mit der kommunale Integrationspolitik an die Erfordernisse einer ,modernen' Verwaltung angepasst werde, mit der aber inhaltlich keine substanziellen Veränderungen verbunden seien (Bommes 2008). In Beiträgen, die dem Integrationsparadigma dezidiert kritisch gegenüberstehen, wurde der ,Paradigmenwechsel‘ bislang kaum diskutiert. Wenn, dann wurden die damit verbundenen neuen Repräsentationen von ,kultureller Vielfalt' und ,Potenzialorientierung' im Rauschen des nationalen Integrationsparadigmas als marginal eingestuft und als Funktionen des neoliberalen ,Aktivierungsimperativs“ im Workfare-Staat, als Ökonomisierung des Sozialen (Lanz 2009) oder als ökonomisierende Ethnisierung kritisiert (Gutiérrez Rodríguez 2003).

Der Beitrag[4] nimmt die einleitende, deutliche Kritik des Frankfurter Konzepts aus Perspektive des Integrationsparadigmas als Hinweis darauf, dass sich ein genauerer Blick auf neue Repräsentationen von (Post-)Migration in aktuellen Strategien kommunaler Integrationspolitik lohnt. Anstatt sie mit einer negativen Kritik an Neoliberalisierungsprozessen kurzzuschließen, richtet sich das Interesse im Folgenden darauf, wie sich staatliche Problematisierungen von (Post-)Migration in der Stadt unter neoliberalen Umständen als (möglicherweise nützliche) Bedingungen für Kämpfe um einen gleichberechtigten Zugang zu Arbeit, Bildung, Politik oder Wohnen in der Stadt darstellen. Die grundlegende These lautet, dass der Staat in der Stadt lernt, (Post-)Migration mit anderen Augen zu sehen: jenseits der nationalen Norm, als konstitutives Moment der ,Vielfalt' von Stadtgesellschaft - (Post-)Migration ist dann keine Kategorie mehr, die prinzipiell über die Teilhabe als Stadtbürger_in bestimmen soll.

Im Kern rekonstruiert der Beitrag also die urban politics of citizenship städtischer Integrationsprogrammatiken. Nach einer einleitenden Diskussion der nationalen bzw. städtischen Bezugspunkte von citizenship wird die in der Bundesrepublik lange dominierende nationale Zurichtung von 
städtischen Politiken herausgearbeitet. Anschließend wird argumentiert, dass die Verlagerung des Integrationsparadigmas auf die Ebene der Stadt die Ordnungslogik des Nationalstaats infrage stellt, weil sich Stadtpolitik heute im Allgemeinen an neoliberalen Rationalitäten orientiert. Programmatisch richten sich Integrationskonzepte wie jenes in Frankfurt dann am Ideal der ,unternehmerischen Stadt' aus und begreifen ihre Einwohner_innen unabhängig von Staatsbürgerschaft und nationaler Zugehörigkeit als zu aktivierende Stadtbürger_innen. Die Genese des Frankfurter Konzepts zeigt abschließend, dass unter diesen neoliberalen Umständen wissenschaftliche Konzepte wie Transnationalismus, super-diversity oder die global city mobilisiert und zum Ausgangspunkt einer Beschreibung urbaner, postnationaler Normalität werden können - an der sich Stadtpolitik in Zukunft auch politisch messen lassen müsste.

Das dem Beitrag zugrundeliegende Projekt[5] analysiert den staatlichen Umgang mit (Post-)Migration in der Stadt aus einer poststrukturalistischen Perspektive. Die Analyse städtischer Programme im Anschluss an die Arbeiten von Michel Foucault (bspw. Kessl/Krasmann 2005, Rosol 2013) wurde um Konzepte der Actor-Network Theory erweitert, um Wissen und Prozesse des Verwaltungshandelns in den Blick nehmen zu können (vgl. Dölemeyer/ Rodatz 2010). Empirische Grundlage sind städtische Dokumente, Experteninterviews mit Verwaltungsmitarbeiter_innen sowie eine dreimonatige teilnehmende Beobachtung im Frankfurter Amt für multikulturelle Angelegenheiten.

\section{Zwischen Stadt und Nation: citizenship und Stadtpolitik}

Die Art und Weise, in der Staatlichkeit in der Moderne zum zentralen Moment der Organisation von Gesellschaft geworden ist, wird seit dem 18. Jahrhundert durch die enge Kopplung zweier Ideen bestimmt: Bürgerschaft und Nation verschmolzen zum Konzept der Staatsbürgerschaft im nationalen Territorialstaat. Der Status der Staatsbürgerschaft regelt nach wie vor im Wesentlichen, wer in welcher Form sein Leben innerhalb der nationalen Grenzen einer Gesellschaft führen kann. In einer Welt globalisierter Wirtschaftssysteme, transnationalisierter Gesellschaften und internationalisierter Politik wird die Funktion dieser Kopplung heute aber zunehmend infrage gestellt. Wenn Städte schon immer Orte waren, an denen um neue Bürgerschaftsrechte gekämpft wurde, dann wird dort heute ihre nationale Form infrage gestellt (Holston/Appadurai 1996: 187f.). Ausgehend von dieser These wurde in den letzten Jahrzehnten der Begriff der urban citizenship diskutiert. Die entsprechende Literatur verwendet einen Begriff von citizenship, der das Verhältnis von formalem Status der Staatsbürgerschaft und der substanziellen Dimension - also der praktischen Wahrnehmung von citizenship in Form sozialer, politischer, ökonomischer und kultureller Teilhabe - differenziert (vgl. Lebuhn 2013: 233).

Für die Diskussion hier ist entscheidend, wie Stadtpolitik aus dieser Perspektive thematisiert wird. Eine erste Variante untersucht sie als Gegenstand von Kämpfen um citizenship: Politische Bewegungen fordern ihre Rechte mit Bezug auf Gender, Sexualität, Ökologie, Migration oder Multikulturalität heute häufig in Städten. Durch die Vielfalt und Hybridität 
von Identitäten, die sich in ihnen sammeln, wird die Artikulation und Durchsetzung neuer Rechte hier wahrscheinlicher (Isin/Siemiatycki 1999: 7f.; vgl. bspw. Garcia 1996, Holston/Appadurai 1996). Entsprechende Kämpfe beschränken sich nicht auf Fragen des Nutzens der Stadt, sondern richten sich gegen jede Form des Ausschlusses vom staatlich zugewiesenen Status oder der Entfaltung von citizenship - (post-)migrantische Kämpfe in diesem Sinne wenden sich gegen die nationale Form solcher Ausschlüsse (vgl. Nyers 2010).

Eine zweite Perspektive beschäftigt sich dezidiert mit der Frage, wie Stadtpolitiken citizenship artikulieren könnten. Wenn Nationalstaaten sich zunehmend den ökonomischen Entscheidungen transnationaler Unternehmen und internationaler Organisationen unterordnen, werden Städte auch als lokalstaatliche Einheiten wichtiger. Hier haben Unternehmen und Organisationen ihre Standorte und zwischen Städten und transnationalen ökonomischen Netzwerken besteht eine direkte wechselseitige Abhängigkeit. Organisationen und Beschäftigte sind auf materielle und soziale Infrastrukturen ebenso angewiesen wie auf öffentliche und private Dienstleistungen. Und Stadtpolitik und -verwaltung müssen sie anbieten, vermitteln und vermarkten (Sassen 1991: 323 ff.). Daran anschließend hat Rainer Bauböck den Vorschlag gemacht, die Autonomie von Städten als Verwaltungseinheiten zu stärken, um den Territorialstaat zu postnationalisieren. Im Zentrum steht dabei eine Idee von urban citizenship, die auf Anwohnerschaft und nicht auf nationaler Zugehörigkeit basiert und auch die Möglichkeit multipler Stadtbürgerschaften und der entsprechenden Wahlrechte innerhalb nationaler Grenzen und darüber hinaus beinhaltet (Bauböck 2003: 139). Interessant wird dieses politikwissenschaftliche Gedankenspiel für die Diskussion hier, weil es Stadtpolitik als Ort der Vermittlung zwischen städtischen Kämpfen um citizenship und der nationalen Verfasstheit von citizenship einführt - urban citizenship als Ausgangspunkt für die Entwicklung einer postnationalen Demokratie, die sich gegen die Imperative nationaler Souveränität und Homogenität richtet und eine Transformation nationaler Identitäten und nationalistischer Ideologien zum Effekt haben soll (ebd.: 157). Aus einer analytischen Perspektive besitzt dieser politische Idealentwurf von urban citizenship allerdings keine Plausibilität. Er formuliert zwar ein plausibles politisch-normatives Ideal, artikuliert aber in keiner Form die machtvollen, verwobenen und widersprüchlichen Rationalitäten, Strategien und Praktiken, die städtisches Regieren empirisch prägen.

Eine dritte Perspektive analysiert urban citizenship als Teil von Strategien und Praktiken städtischen Regierens. So untersucht Nicolas Rose (2000) aktuelle urban politics of citizenship vor dem Hintergrund neoliberaler Transformationen urbaner Gouvernementalität seit den 1980er Jahren. Den Kern dieser Transformation begreift Rose als Veränderung der staatlichen Problematisierung der Ordnung der Stadt. In der ersten Hälfte des 20. Jahrhunderts habe der Traum einer planbaren, rationalen Ordnung der Stadt die Regierung städtischen Lebens angeleitet, der im Wesentlichen den biopolitischen Ordnungsmustern des Nationalstaats folgte (vgl. Osborne/ Rose 1999: 745). Heute hätten sich hingegen die Regierungsweisen der Stadt, das Wissen, auf das diese sich beziehen, und damit die Problematisierungen städtischen Lebens vervielfacht. Ziel der entsprechenden Programme sei es, Ordnung aus der Vielfalt städtischen Lebens zu schöpfen: „These [programs] 
seek new ways of harnessing the forces immanent within urban existence: they dream of a city that would almost govern itself" (Rose 2000: 96). Mit diesen Transformationen ist eine Verschiebung der urban politics of citizenship verbunden, so Rose weiter:

"This is not a relation of citizen and community in terms of blood, descent, lineage, tradition, fixity, mechanical solidarity and the like, but a relation of identification. [...] Citizenship - ceasing to be a kind of ,posssesion' or simple right of persons - has taken on a relational form. Citizenship is as much a capacity to act in relation to the particular circumstances of one's environment, as well as in relation to others, as it is a right' conferred by the state. If the city is again central here it is in that $[. .$.$] - insofar as it is a concrete, localized space - the city can take$ over from the state as the primary reference point of citizenship. This transformation from citizenship as possession to citizenship as capacity is embodied in the image of the active and entrepreneurial citizen who seeks to maximize his or her lifestyle through acts of choice, linked not so much into a homogeneous social field as into overlapping but incommensurate communities of allegiance and moral obligation." (Ebd.: 98f.)

Folgt man dieser Argumentation, stellt sich die Stadt auch aus einer analytischen Perspektive auf Strategien und Praktiken städtischen Regierens als Ort einer Transformation der nationalen Organisation von Zugehörigkeit dar. Diese Beobachtung einer Verschiebung von urban politics of citizenship wird im Folgenden herangezogen, um zwei Artikulationen von citizenship in Rationalitäten des städtischen Umgangs mit (Post-)Migration in der Bundesrepublik zu differenzieren. Dabei zeigt sich zunächst, dass in der Vergangenheit hegemoniale Strategien (Post-)Migration im Namen der Nation problematisiert haben. Die Bürger_innen, für die sie sprechen und handeln, sind Staatsbürger_innen, deren Gemeinschaft nach dem in Deutschland gültigen Prinzip des ius sanguinis ganz wörtlich und konkret „in terms of blood, descent, lineage, tradition, fixity, mechanical solidarity and the like“ (ebd.) zu verstehen ist.

\section{Stadtpolitik im Namen der Nation}

Deutsche Stadtpolitiken haben das Verhältnis zu ihrer (post-)migrantischen Bevölkerung lange Zeit ausschließlich in Begriffen der Staatsbürgerschaft praktiziert - für die Bundesrepublik zutreffender: in Begriffen des Ausschlusses von der Staatsbürgerschaft. Denn im ,Nichteinwanderungsland' war die Möglichkeit zur Einbürgerung mit wörtlichem Bezug auf ,blood, descent, lineage, tradition' prinzipiell nicht vorgesehen.[6] Aufenthaltsberechtigte Ausländer_innen wurden deutschen Staatsbürger_innen zwar in vielen Punkten gleichgestellt. Allerdings konnten diese Rechte durch Verwaltungsmaßnahmen im Aufenthalts- und Arbeitserlaubnisverfahren praktisch jederzeit entzogen werden, wobei die ,Zweckmäßigkeitserwägung' der Verwaltung beispielsweise bei der Durchsetzung des ,Inländerprimats‘ durch einen gesetzlich fixierten breiten Ermessensspielraum der Verwaltung abgesichert wurde (Dohse 1981: 301f.). Das Ausländerrecht steht in seiner Genese aus der Ausländerpolizeiverordnung von 1938 (bis 1965 in Rechtsfolge gültig) 
für eine Traditionslinie staatlichen Zugriffs, der (Post-)Migration in erster Linie als Problem der öffentlichen Sicherheit und Bedrohung der nationalen Solidargemeinschaft konstruiert (Karakayali/Tsianos 2002: 252) - diese Problematisierung als Gefahr für deutsche Arbeitsplätze, die öffentliche Ordnung und die Identität Deutschlands dominierte über alle Konjunkturen des deutschen Migrationsregimes hinweg (Green 2006: 115).

Auch für Städte im ,Nichteinwanderungsland“ stellte sich (Post-)Migration dann lange praktisch ausschließlich als Ordnungsproblem für die staatsbürgerliche Solidargemeinschaft dar. Diese Rationalität artikulierte sich deutlich, als sich die (Post-)Migration in der Bundesrepublik im Laufe der 1960er Jahre verstärkt der ökonomischen Funktionalisierung des Gastarbeiterregimes entzog, indem die ,Gastarbeiter ' eine Normalisierung ihrer Lebensverhältnisse außerhalb von Firmenbaracken oder Gastarbeiterwohnheimen und in einem sozialen Lebensumfeld anstrebten. Staatliche Strategien verarbeiteten diese Entwicklung im Kontext der ökonomischen Krise auf nationaler Ebene durch den Anwerbestopp (Karakayali/Tsianos 2002: 254). Die bereits bestehende (post-)migrantische Bevölkerung in den Städten wurde in der Folge als „Massierung von Ausländern in den Verdichtungsgebieten“ zum „Kernproblem der gesamten Ausländerbeschäftigung “erklärt (Happe 1975, zit. n. Münch 2010: 276). In Verbindung mit dem nicht vorhergesehenen und als unkontrollierbar wahrgenommenen Familiennachzug wurde eine städtische Problematisierung von (Post-)Migration als, Überlastung' der Infrastruktureinrichtungen und des sozialen Gefüges hegemonial (Karakayali/Tsianos 2002: 255). Konkret verband man mit der (post-)migrantischen Bevölkerung praktisch alle zeithistorischen Probleme der Städte: „Wohnungsmangel, Infrastrukturdefizite, Originalitätsverluste, Integrationsprobleme, Überfremdungsangst und Umweltschäden“ wurden „mit den rasch ansteigenden Ausländerzahlen in Zusammenhang gebracht“ (Ganser 1974: 33).

Eine ,Integration' der (post-)migrantischen Bevölkerung wurde - wenn überhaupt - nur auf Zeit und im Namen des Schutzes der autochthonen Bevölkerung der Städte konzipiert und stellte sich als ausländerpolitisch gerahmte, soziokulturelle staatliche Fürsorge ein, die häufig von Strategien der Rückkehrförderung begleitet wurden (Lanz 2009: 105). In der Bundesrepublik änderte auch die in den 1980er Jahren aufkommende Multikulturalismusdebatte wenig an dieser grundsätzlichen Hegemonie - über weite Strecken blieb sie auf einen „feuilletonistischen und folkloristischen Einschlag" reduziert und schrieb sich kaum in staatliche Politiken ein (Aumüller 2009: 198). Im Allgemeinen waren die 1990er Jahre eher durch eine Verstärkung der Rhetorik des ,Nichteinwanderungslandes' geprägt: Die rassistischen Pogrome und Morde unter anderem in Rostock-Lichtenhagen und Mölln gingen einher mit politischen Debatten um ,Flüchtlingsflut und ,Asylmissbrauch“ und führten schließlich zur faktischen Abschaffung des Asylrechts im sogenannten Asylkompromiss (Reimer 2013: 32). Die Reformen des Ausländerrechts ab Ende der 1990er Jahre bis zur Einführung des neuen Zuwanderungsrechts im Jahr 2005 wurden von Debatten um eine ,deutsche Leitkultur ' (Hentges 2002) und der wachsenden Dominanz eines antimuslimischen Rassismus flankiert (Attia 2007).

So waren mit den Reformen des Zuwanderungs- und Ausländerrechts zwar auch neue Selbstbeschreibungen der Bundesrepublik als von kultureller 
Vielfalt geprägtes Land, internationaler Wirtschaftsstandort und weltoffene Nation verbunden. Im Kern beinhaltete dies aber keine systematische Öffnung, sondern die politische Gestaltung des Umgangs mit der bestehenden (post-)migrantischen Bevölkerung. Das deutsche Selbstverständnis verschob sich also weniger zum ,Einwanderungsland' als vielmehr zum ,Integrationsland' und konservierte damit die Tradition eines rassistischen Misstrauens gegenüber einem großen Teil seiner (post-)migrantischen Bevölkerung (Meier-Braun 2013: 18ff.). Im Kern der systematischen Einführung des Integrationsparadigmas stand die Fortschreibung eines essentialistischen Kulturverständnisses - mit dem in diesem Kontext etablierten Begriff des ,Migrationshintergrundes “ wird die „nicht-deutsche kulturelle und nationale Herkunft bis zum Nimmerleinstag" fortgeschrieben und daran ein zu bearbeitendes Integrationsdefizit gekoppelt (Hess/Moser 2009: 13). Mit dieser spezifischen Aufforderung zum ,lebenslangen Lernen' wird auch die Anpassung der Integrationspolitik an den Umbau der Bundesrepublik vom Welfare- zum Workfare-Staat deutlich, dessen zentrales Prinzip das ,Fordern und Fördern' aller - jedoch besonders der als defizitär definierten - Subjekte ist (ebd.: 11).

Auf der städtischen Ebene wurden neuerliche Schreckensmeldungen der scheiternden Integration nun zunehmend mit dem, islamischen Kulturkreis in Verbindung gebracht - die Rede war von ,ethnischen Enklaven` und ,Parallelgesellschaften', von ,Familienclans', ,Ehrenmorden' und ,Zwangsehen (ebd.: 12). Insofern verwundert es wenig, wenn Stefan Lanz am Beispiel Berlins auch aktuell noch einen differenziellen Integrationsdiskurs als prägend identifiziert, der im Wesentlichen dem bislang diskutierten „traditionellen deutschen Modell“ folgt und Integration in den Dienst des „,historisch hegemonialen Konzepts der deutschen Nation als Abstammungs- und Kulturgemeinschaft“ stellt (2009: 109). Entsprechende stadtpolitische Diskurse fungieren bis heute in „Verteidigung der Gesellschaft“ (Foucault 2001), als lokalstaatliche Durchsetzung der rassistischen Solidargemeinschaft im „national-sozialen Staat“ (Balibar 2001: 9, vgl. Rodatz 2012: 74ff.)

\section{Integrations- und Vielfaltspolitik in der ,unternehmeri- schen Stadt ${ }^{6}$}

Im Folgenden wird argumentiert, dass aktuelle integrationspolitische Strategien sich als urban politics of citizenship verstehen lassen, dievon der bislang geschilderten Konzeption von Stadtpolitik im Namen der Nation abweichen. Ausgangspunkt der Überlegungen ist die Neueinführung sogenannter strategischer Integrationskonzepte - mittlerweile verfügen 9o Prozent der deutschen Großstädte über entsprechende Dokumente (Gesemann et al. 2012: 40). Diese erfolgreiche Mobilisierung von Städten zu einer konzeptionellen Neuaufstellung ihrer Integrationspolitiken wurde durch Standardisierung und interkommunalen Austausch organisiert. Sie lässt sich als konzertierte Verlagerung von Integrationspolitik auf die Ebene Stadt verstehen, in deren Verlauf eine Reartikulation des Integrationskonzepts entlang neoliberaler Rationalitäten der „unternehmerischen Stadt“ (Harvey 1989, s. u.) zu beobachten ist. Zwar ist der interkommunale Austausch weder an sich neu noch notwendig neoliberal (Clarke 2012). Jedoch wird er im Feld kommunaler 
Integrationspolitiken (wie in anderen Bereichen auch) durch Technologien des Vergleichs, des Benchmarkings, der Identifikation von Best Practices und deren Vermarktung als „fast policy regime“ (Peck 2001: 452) strukturiert, deren Ergebnis häufig die Implementation neoliberaler Politiken ist (Pütz/ Rodatz 2013: 176).

Kern der Mobilisierung von Integrationskonzepten ist die Überführung von Integrationspolitik in neue Steuerungsmodelle - Integrationspolitik soll „im Design moderner Verwaltung“ praktiziert werden, also als Integrationsmanagement auf Grundlage strategischer Konzepte, deren Etablierung durch „Evaluation und Monitoring auf der Grundlage von definierten Indikatorensets“ überwacht wird (Bommes 2008: 164). Die zugrunde liegenden Steuerungsideen verlaufen im Wesentlichen entlang der Linien allgemeiner Verwaltungsreformen. In der Bundesrepublik werden städtische Bürokratien seit den 1990er Jahren mit den Schlagworten Ergebnisorientierung, Globalbudgets, Leistungsmessung, Wettbewerb und Controlling reorganisiert. Anstatt politische Ziele in die Zuteilung von Personal-, Finanz- und Sachmitteln zu übersetzen und die Verwaltung ihr Werk verrichten zu lassen (Inputsteuerung), werden die zu erreichenden Ergebnisse in Form von strategischen Zielen und Produktkatalogen formuliert und der Weg der Realisierung dieser Ergebnisse durch ein neues Berichtswesen, Leistungsindikatoren und darauf bezogene Budgets überwacht. Weil die öffentliche Verwaltung dabei einer unternehmerischen Logik unterworfen wird und unter Kostendruck um Investitionen, Arbeitsplätze und Steuereinnahmen konkurriert, stellen diese Reformen keine ,neutralen Modernisierungsmaßnahmen dar. Zentraler Effekt dieser Logik ist, dass politische Fragen auf Kostenüberlegungen reduziert werden - sie lässt sich insofern als „neoliberale Neuordnung städtischer Verwaltungen“ verstehen (Silomon-Pflug/Heeg 2013, vgl. Lebuhn 2010).

Der Kontext solcher Steuerungsmodelle ist aus politökonomischer bzw. regulationstheoretischer Perspektive im Anschluss an David Harvey (1989) auf den Punkt gebracht worden. Sie sind einerseits durch den Abbau staatlicher Interventionsmöglichkeiten (Deregulierung), fiskalische Restriktion (Austerität) und den Rück- bzw. Umbau sozialer Sicherungssysteme gekennzeichnet. Andererseits beinhalten sie den Aufbau neuer Strategien, mit denen staatliche Gestaltungsmacht auf lokaler Ebene wiedererlangt werden soll. Städte würden in der Folge nicht nur als subnationale staatliche Einheiten in eine neue, relative Autonomie gedrängt, sondern als ,unternehmerische Stadt ‘ für die lokale ökonomische Entwicklung verantwortlich gemacht und einem neuen, nationalen und transnationalen Standortwettbewerb ausgesetzt (vgl. Brenner 2003: 214). Aus gouvernementalitätsanalytischer Perspektive sind die mit diesen Neuordnungsprozessen verbundenen Regierungstechnologien als Implementation neoliberaler Rationalitäten auf der städtischen Ebene hinsichtlich der damit verbundenen Macht- und Subjektivierungsformen beschrieben worden (bspw. Kessl/Krasmann 2005, Rosol 2013; konkret zur ,unternehmerischen Stadt' Schipper 2013).

Im Anschluss an diese Argumentationen lässt sich zeigen, dass aktuelle städtische Integrationskonzepte jene urban politics of citizenship in der unternehmerischen Stadt artikulieren, die oben mit Rose vorgestellt wurden. Die Anbindung an die Funktionsbedingungen der unternehmerischen 
Stadt lässt sich in vier zentralen Strategien identifizieren, die wir an anderer Stelle empirisch vorgestellt haben (vgl. Pütz/Rodatz 2013): Die Konzepte problematisieren die vergangene ,Defizitorientierung' und konzipieren (Post-)Migration als konstitutiven Bestandteil der Stadt, dessen schöpferische Kraft freigelegt werden müsse (1); mit diesem Ziel müsse Integrationspolitik von einer, ausländerpolitischen' Sonderaufgabe zu einer städtischen Querschnittsaufgabe entwickelt werden (2); in der Konsequenz gelten dann der Ausbau kommunalpolitischer Integrationsnetzwerke (3) und die Fokussierung von Integrationspolitik auf den Sozialraum als inhaltliche Voraussetzungen einer erfolgreichen Steuerung (4). Entgegen der These, dass die Anpassung städtischer Integrationspolitiken an das „Design moderner Verwaltung" keine wesentliche inhaltliche Neuausrichtung mit sich bringe (Bommes 2008: 164), lässt diese sich entlang der Strategien der Potenzial-, Netzwerk- und Sozialraumorientierung als Artikulation von aktivierenden Macht- und Subjektivierungsformen analysieren.

Wenn den Kommunen als unternehmerische Stadt eine neue Verantwortung für die Aktivierung ihrer Bürger_innen unter der Prämisse des ,Forderns und Förderns' zugesprochen wird, dann äußert sich dies als Subjektivierungsverhältnis, das als neosoziale Responsibilisierungsstrategie beschrieben worden ist: Soziale Rechte werden damit an die Selbstverantwortung der Individuen geknüpft. Entsprechende Strategien suchen ihr Klientel im Rückgriff auf sein soziales und ökonomisches Kapital zur aktiven und ,selbstverantwortlichen' sozialen, ökonomischen, kulturellen und politischen Gestaltung des eigenen Lebens zu aktivieren (Kessl/Krasmann 2005: 234). Als Regierungsstrategie knüpfen sie den erweiterten Raum zur Selbstentfaltung ihrer Subjekte an die Erwartung, dass dieser selbstständig in einer Weise genutzt wird, die zumindest keine Kosten verursacht und im besten Fall ,produktiv ist. Die Strategien führen also nicht weniger, sondern in einer Weise, die die Notwendigkeit sozialpolitischer Interventionen zu minimieren sucht. Auch der Staat dieser Strategien ist nicht ,weniger', sondern ,anders': Ein Staat, der die Selbstführungskompetenzen seiner Bürger_innen fördert, kontrolliert und im Fall der Abweichung sanktioniert (Bröckling et al. 2000: 26).

Diese Strategie übersetzt sich integrationspolitisch zunächst in die Potenzialorientierung - und ist mit Blick auf die Vergangenheit einer Regierung der Stadt im Namen der Nation voraussetzungsvoll. Strategien, die auf die Stärkung der Stärken ihrer Klientel zielen, müssen schließlich zunächst einmal lernen, diese Stärken auch zu erkennen. Die Potenzialorientierung äußert sich in den städtischen Integrationskonzepten dann auch nicht nur als neue Perspektive (Pütz/Rodatz 2013: 117), sondern als dezidierte Kritik der ,defizitorientierten' stadtpolitischen Vergangenheit. Selten wird diese Bedeutung so deutlich expliziert wie im Beispiel des Konzepts der Stadt Leipzig:

„Während lange Zeit in der Bundesrepublik aus einer eher problemund defizitorientierten Sichtweise heraus agiert und dementsprechend die Integrationsarbeit im Sozialressort verortet wurde, setzt sich in den letzten Jahren mehr und mehr die Erkenntnis durch, dass Integrationspolitik angesichts der demografischen Realität und der Globalisierung der Wirtschaft auch die Potenziale von Migration 
benennen, entsprechend würdigen und als Bereicherung für die kommunale Entwicklung verstehen sollte.“ (Stadt Leipzig 2006: 9f.)

Die Strategie der Potenzialorientierung artikuliert eine veränderte politische Repräsentation von (Post-)Migration als Teil der zu aktivierenden Bürger_innen der unternehmerischen Stadt. Besonders deutlich artikuliert sich diese Strategie in Städten, die sich selbst als globaler Standort vermarkten und dabei ihre ,kulturelle Vielfalt' als funktionale Notwendigkeiten und Zeichen einer erfolgreichen, transnational vernetzten städtischen Ökonomie und Gesellschaft repräsentieren:

„Vielfalt bedeutet Stärke - dieser Grundsatz einer modernen Unternehmenskultur gilt besonders in Berlin. Migrantinnen und Migranten tragen zu dieser Stärke wesentlich bei. [...] Berlin ist nicht nur im Kampf um Investitionen ein Global Player, sondern auch im Wettbewerb um kluge und aktive Menschen." (Stadt Berlin 2007: 1)

Nicht alle untersuchten Konzepte nutzen die Semantik der,Vielfalt' so zentral oder koppeln sie so eindeutig an die neoliberale Strategie des „DiversityManagements“ und dessen enge „ökonomische Logik“ (Aretz 2004). Aber alle untersuchten Konzepte leiten den Handlungsbedarf nicht mehr maßgeblich aus den vermeintlichen Defiziten von (Post-)Migrant_innen ab, sondern aus ihrem Potenzial für die Selbsteingliederung und die Entwicklung der Stadt insgesamt.

Auch in der Strategie der Netzwerkorientierung zeigen sich Steuerungstechniken der Responsibilisierung. Der grundsätzliche Kontext dieser Strategie wird für die Regierung der Stadt unter dem Begriff urban governance gefasst. In Abgrenzung zu hierarchischen Steuerungsmodellen (urban government) etabliert sich demnach seit den 1980er Jahren im Rückgriff auf Effizienz- und Innovationsargumente eine neue Arbeitsteilung des Regierens in Form strategisch gesteuerter netzwerkförmiger Abläufe. Im Zuge dieser Logik sollen durch strategische Anreize autonome ,Partner des Regierens hergestellt und mobilisiert werden, mit dem mittelbaren Ziel, bestehende Ressourcen vor Ort im Sinne einer Selbstintegration zu nutzen (Rose 2000: 96f.). Vor diesem Hintergrund beziehen sich Verwaltungen positiv auf die soziale, ökonomische oder kulturelle Vielfalt der Stadtgesellschaft, begreifen auch (post-)migrantische Differenzen als Organisationsressourcen und fördern die Selbstorganisation. In den Fokus der integrationspolitischen Netzwerkorientierung geraten dann auch (post-)migrantische Organisationen (bspw. Kulturvereine und religiöse Zuwanderergemeinden), deren gesellschaftliche Partizipation im Namen der Multiplikation der Selbstführungskräfte gefördert werden soll (Pütz/Rodatz 2013: 171).

Die Strategie der Sozialraumorientierung von Integrationspolitik lässt sich als neosoziale Form der Territorialisierung begreifen, die ebenfalls auf eine - räumlich spezifische - Aktivierung produktiver Netzwerke oder Communitys setzt (Kessl/Krasmann 2005: 236). Die Folge für die Regierung von (Post-)Migration zeigt sich in den Integrationskonzepten zunächst als Aufgabe der pauschalen raumordnungspolitischen Problematisierung von (Post-)Migration. Ziel der Stadtpolitiken im Namen der Nation war eine ,ethnisch' durchmischte Stadt; als Zeichen ihres drohenden Scheiterns 
wurden deshalb stets sichtbar (post-)migrantisch geprägte Viertel als ,Ausländerkonzentrationen', ,Ghettos' oder ,Parallelgesellschaften' ausgemacht (Ronneberger/Tsianos 2009). In den Integrationskonzepten wird die Kritik an dieser Zielstellung der Politik zum Ausgangspunkt neuer Interventionsformen (vgl. Pütz/Rodatz 2013: 171f.). Dabei wird Segregation als Mittel zur Entfaltung einer produktiven, aber riskanten lokalen Vergemeinschaftung konzipiert. In dieser Vorstellung bergen also (post-)migrantische Gemeinschaften, etwa in Form ,ethnischer Netzwerke', einerseits Ordnungspotenzial, das für soziale, kulturelle und politische Partizipation nutzbar gemacht werden kann. Auf der anderen Seite werden sie weiterhin als drohende ,Parallelgesellschaften' und Formen einer unproduktiven Vergemeinschaftung problematisiert. Positive wie negative sozialräumliche Repräsentationen von (Post-)Migration werden dabei grundsätzlich an die Aktivierungsstrategien der quartiersbezogenen Stadtteilentwicklung angeschlossen: Die Bewohner_innen der Stadtteile sollen selbst aktiv werden und ,Verantwortung für ihre Lebenssituation übernehmen. Gefordert und gefördert wird dann, Teil einer sichtbaren lokalen Gemeinschaft zu sein, die zur Wertschöpfungskette der Stadt beiträgt. Andernfalls wird man als unproduktive oder nichtvernetzte „Anti-Bürger_in“ (Rose 2000: 103) zur Gefahr für eben diese Wertschöpfungskette erklärt (vgl. Rodatz 2012: 191).

Zusammenfassend lassen sich die in den Konzepten formulierten Strategien - im Kontrast zu den Ausführungen im zweiten Abschnitt - als Verschiebung des städtischen Umgangs mit (Post-)Migration und damit als veränderte urban politics of citizenship beschreiben. Die Programme inszenieren (Post-)Migration als konstitutives Moment von Stadtgesellschaft und richten sich damit an einem Zugehörigkeitsmodell aus, das sich mit Rose als Stadtbürgerschaft verstehen lässt. Für die unternehmerischen Subjekte dieser Programme ist es ,rational', wenn sie ihre spezifischen (auch: (post-) migrantischen) Ressourcen nutzen, solange sie damit zur Ordnung der Stadt beitragen. In diesem Sinne verschiebt sich die Beziehung von citizenship und (Post-)Migration in der Stadt: von der Bedeutung als Abweichung von der nationalen Gemeinschaft der Staatsbürger_innen hin zum Bezug auf das Ideal der aktiven Stadtbürger_in, um deren (Selbst-)Führung sich die Programmatik dreht. Die unternehmerische Stadt ist nicht mehr die Stadt der rassistischen Solidargemeinschaft, in der es den sozialen Frieden der deutschen Abstammungsgemeinschaft vor der (Post-)Migration zu schützen gilt - es ist die Stadt der zu Ertüchtigenden. Die Demarkationslinie dieser Programme verläuft nicht mehr prinzipiell entlang ethnisch-nationaler Kategorien, sondern in Begriffen der Aktivierbarkeit, und insofern hat die pauschale Unterscheidung zwischen Deutschen und (Post-)Migrant_innen hier keine Funktion mehr.

\section{Was Neoliberalismus auch kann: No integration!}

Wenn diese aktuellen Strategien städtischer Integrationspolitik Zugehörigkeit jenseits der essentialistischen Linien des nationalen Narrativs von Blut und Abstammung, Tradition und Kultur (Rose) artikulieren, dann nicht, um die Stadt als „homebase for cosmopolitan democracy“ zu entwickeln, wie es Bauböck (2003: 157) vorgeschlagen hat. Sie tun es vielmehr aus einer 
ökonomischen Rationalität heraus, in deren Zentrum das Bild aktiver und unternehmerischer Stadtbürger_innen steht, die zur Prosperität der Stadt beitragen sollen. Dennoch richtet sich die Analyse hier abschließend am Beispiel des Frankfurter Konzeptes nicht auf neoliberale Integrationspolitik als „restriktives, ökonomistisch verengtes Modell“ (Lanz 2009: 119), sondern auf jene Momente, in denen entsprechende städtische Strategien „über Fragen von ,Fördern“ und ,Fordern“ weit hinaus“ gehen (Stadt Frankfurt 2011: 9). Dabei steht die Frage im Mittelpunkt, inwiefern der neoliberale Kontext der unternehmerischen Stadt selbst dazu beitragen kann, eine Konzeption von Integrationspolitik als „Vielfaltspolitik“ hervorzubringen, die „Einwanderer als politische Bürger adressiert“ (Lanz 2009: 119). Lanz grenzt ein solches „emanzipatorisches Modell“ (ebd.) dezidiert von der dominierenden Lesart im Zeichen des „neoliberal turn“ ab, in der „Integrationspolitik [...] nicht mehr aus einer sozialpolitischen Perspektive betrieben, sondern als Investition in das unternehmerische Subjekt ,Einwanderer" verstanden“ wird (ebd.: 106).

Ausgangspunkt ist die Überlegung, dass die Kritik am Neoliberalismus häufig von der Tradition der nationalen Form des Sozialen und deren rassistischen Effekten abstrahiert, wenn sie das Soziale gegen die Ökonomisierung verteidigt. Wenn also (berechtigterweise) kritisiert wird, dass mit dem Übergang zum Workfare-Staat der sozialpolitische Ausgleich eingebüßt werde, gerät in Vergessenheit, dass dieser Ausgleich eine nationale Solidargemeinschaft inszenierte, die es vor (Post-)Migration zu verteidigen galt. Und wenn (berechtigterweise) kritisiert wird, dass die responsibilisierenden Strategien in der neoliberalen Stadt die sozialen Verwerfungen durch Ökonomisierung und Privatisierung verschleierten, dann wird übersehen, dass damit erstmals nicht nur die ,ethnisch' Deutschen als Bürger_innen der Stadt angesprochen werden (vgl. Rodatz 2012: 91ff.). Zugespitzt formuliert: Während Stadtpolitiken im Wohlfahrtsstaat die nationale Verteidigung der Gesellschaft betrieben, wenn sie die soziale Infrastruktur vor der ,ausländischen' Bevölkerung zu schützen suchten, steht heute mit dem neoliberalen Funktionswandel von sozialem Ausgleich im Allgemeinen und mit der Aufwertung der Stadt als unternehmerische Einheit im internationalen Wettbewerb die nationale Form dieser Politiken zur Disposition - und damit eröffnet der neoliberal turn selbst politische Spielräume für eine ,kosmopolitische“ Entfaltung von citizenship. Diese Spielräume in den Fokus zu stellen, folgt hier dem Vorschlag von James Ferguson, die Allgegenwart von Neoliberalisierungsprozessen nicht länger nur mit der ständigen Wiederholung ihrer (zweifellos richtigen) Kritik zu beantworten, sondern zu fragen, was diese Umstände für die Durchsetzung „progressiver“ Politiken bedeuten können (2009: 173). Ein solcher Blick könnte lohnenswert sein, denn Neoliberalisierungsprozesse sind häufig überraschender, als es der Begriff (in vielen ,kritischen“ Analysen) vermuten lässt: „[T]his has been associated with an evolving, experimental policy repertoire, beset by contradictions“(Peck/Theodore 2010: 173).

Eine postnationale Artikulation aktivierender Anrufungen im Zeichen der Potenzialorientierung auf Ebene der Stadt müsste sich dann von den Mustern des Integrationsparadigmas lösen und stattdessen dezidiert postnationale urban politics of citizenship beinhalten. Denn im Kontext des nationalen Integrationsparadigmas „erfahren Migrantinnen und Migranten trotz des 
scheinbaren Bestrebens des neo-liberalen Staates, sie auf der ökonomischen Ebene zu integrieren, immer wieder eine Anrufung als subjectless objects“ und „werden als die differenten defizitären Anderen markiert“ (Gutiérrez Rodríguez 2003: 174f.).

Im Mittelpunkt des Frankfurter Konzepts steht eine Repräsentation von (Post-)Migration als potenziell schöpferische Kraft, die auf die Stadtgesellschaft und die Stadtentwicklung in ökonomischer und sozialer Hinsicht bezogen wird und dabei ein Verhältnis wechselseitiger Prägung formuliert. Am deutlichsten artikuliert sich diese Konnotation darin, dass das Attribut der „Vielfalt als Tatsache“ (Stadt Frankfurt 2011: 15) nicht nur auf bestimmte Bevölkerungsgruppen bezogen, sondern als konstitutives Merkmal der städtischen Gesellschaft insgesamt begriffen wird. Unter diesen Umständen, so das Konzept weiter, verändert sich auch die Bedeutung von ,Integrationspolitik als einer „Politik der Vielfalt, die die urbane Realität ernst nimmt“ (ebd.: 12):

„Eine Politik, die wirklich integrieren will, kann nicht mehr nur mit den üblichen Kategorien ,ethnischer oder ,kultureller Einwanderergruppen arbeiten, sondern muss die viel komplexere Realität der gesamten Frankfurter Stadtgesellschaft im Blick haben: ein Neben- und Miteinander sich überkreuzender, sich verändernder, sich weiter ausdifferenzierender oder auch mischender und neu bildender Milieus." (Ebd.: 15)

Das Konzept bezieht die Konzeption dieser „komplexen Realität“ aus einer wissenschaftlichen Expertise, die im Entwurfsprozess eine „Untersuchung der Realität unserer Stadtgesellschaft aus vielfältigen und oft transnationalen Beziehungen und Gruppen“ vorgestellt hatte (ebd.: 10). Diese Expertise mobilisierte ausschließlich wissenschaftliche Konzeptionen, deren kleinster gemeinsamer Nenner ist, dass sie grenzüberschreitende Migrationen als konstitutives Moment von Vergesellschaftung verstehen (s. u.). Die Genese dieser Perspektive wird im Folgenden aus der Feldforschung im Frankfurter Amt für multikulturelle Angelegenheiten (AmkA) mit Blick auf die spezifische Artikulation von citizenship im Frankfurter Integrationskonzept rekonstruiert.

Nach Darstellung der für die Entwicklung des Konzepts anfangs zuständigen Mitarbeiterin eröffnete auch in Frankfurt der ,Reformdruck' aus dem interkommunalen Vergleich den Prozess, allerdings mit Spielraum für Abgrenzung zu verbreiteten integrationspolitischen Modellen und Vorbildern anderer Städte:

„Ab 2007 war also klar, da besteht ein irrer Handlungsbedarf, was Frankfurt anbelangt. [...] Verschiedene Stiftungen, allen voran die Bertelsmann Stiftung, haben in dieser Zeit zusammen mit den Ministerien begonnen, Instrumente zu promovieren: [...] gesteuerte Integration, durch Konzepte und Monitoring. [...] Frankfurt hat da lange nichts davon gemacht. [...] Zu dem Zeitpunkt gab es ja immer so ein gewisses Schwanken zwischen Integration im Sinne von Assimilation und Vielfaltsgeschichten, also immer in unterschiedlichen Ausprägungen, je nach Stadt, je nach Stadtpolitik. Und man hat immer unglaublich gerungen um diesen Integrationsbegriff und diese 
Esser-Definitionen[7] standen immer sehr im Vordergrund bei vielen Städten. Und ich habe ein Plädoyer dafür geschrieben, dass wir hier in Frankfurt einen ganz anderen Ansatz brauchen, einen ganz anderen Blick auf die Stadt. Ich wusste ja auch, dass in der Migrationsforschung seit Langem auch völlig andere Perspektiven entwickelt worden waren, die hier überhaupt noch nicht angekommen waren in der deutschen Integrationspolitik.“ (I 5 13/o1)

Zwei Referenzketten sollten den weiteren Prozess also prägen: eine spezifische Tradition Frankfurter Integrationspolitik seit Gründung des AmkA und neue Entwicklungen der Migrationsforschung.

Das Verständnis einer atypischen Tradition der Frankfurter Integrationspolitik handelt von der unzeitgemäßen Gründung des AmkA und von seinem politischen Selbstverständnis:

„Die Gründung des Amts fiel ja in eine Zeit, die von einem starken Rechtstrend geprägt war. Auch in der Stadtpolitik hieß es: ,die gehören hier nicht her', und es wurde irgendwie viel dem Volk nach dem Mund geredet und so weiter. [...] Es war ja genau die Zeit, als es überall Übergriffe gegen Flüchtlinge gab und wir hatten eine Höllenangst, dass hier irgendwas in Frankfurt passiert. [...] Und in diese Zeit kam dieses Multikulti-Amt und das hat ja auch wie eine Bombe eingeschlagen.“ (I 5 13/01)

Das AmkA wurde 1989 von den Grünen in den Koalitionsverhandlungen mit der SPD durchgesetzt. Im vorausgehenden Wahlkampf hatte nicht nur die NPD mit rassistischen Positionen erfolgreich Stimmen einwerben können. Auch die CDU hatte die ,Ausländerfrage in den Mittelpunkt des Frankfurter Wahlkampfs gestellt: Man müsse „zur Kenntnis nehmen, daß es in der Bevölkerung den ,eindeutigen Wunsch' nach einer Begrenzung des Zuzugs von Ausländern und einer Beendigung des Mißbrauchs des Asylrechts“ gebe (Mick 1989). In diesem Kontext entstand das AmkA als städtische Einrichtung, deren einzige Aufgabe es sein sollte, die Interessen der (post-) migrantischen Bevölkerung innerhalb der Stadtverwaltung zu vertreten (Aybek/Straßburger 2009, Leggewie 2011). Das Amt trieb durch ein „persuasives Programm“ den Perspektivwechsel der Stadt auf ihre (post-)migrantische Bevölkerung voran und implementierte in diesem Sinne Politiken, zu denen sehr früh und zentral auch Antidiskriminierungsarbeit gehörte (Leggewie 2011). Trotz berechtigter Kritik an technisierenden und paternalistischen Praktiken des Amts ließen sich bereits diese Entwicklungen vor dem Hintergrund des fortbestehenden faktischen Ausschlusses vom formalen Status der Staatsbürgerschaft als substanzieller Ausbau von urban citizenship verstehen (Welz 1998: 44, vgl. Mestre Vives 1998).

Die Gründung des Amts lässt sich insofern auch als Kontrapunkt zum nationalen Zeitgeist und damit zur Natur des deutschen Staats verstehen. Und „eigentlich ist das, worum wir damals gerungen haben, genau das Gleiche, worum man heute immer noch ringt“ (I 5 13/01) - das AmkA wird in diesem Narrativ als Versuch gewertet, die staatliche und politische Negation der (post-) migrantischen Gesellschaft zu durchbrechen und zumindest für Frankfurt die Strukturen an das anzupassen, was da draußen schon längst gelebt würde: „Wir 
sind Frankfurter, wir gehören hierher" (I 5 13/o1). Der Griff zum Konzept des Multikulturalismus sei aus dieser Perspektive heraus der Versuch gewesen, neue Begriffe für eine adäquate Selbstbeschreibung der Stadtgesellschaft zu finden. Aus heutiger Perspektive erschienen die vergangenen Konzeptionen des AmkA aber als Versuch, der auch in der Praxis immer wieder offenbarte, „wie hilflos wir mit den Begriffen hinterherhinken - bis heute - hinter dem, was passiert“(I 513/O1). In den 20 Jahren seit Gründung des AmkA hätten sich aber „von der Wissenschaftsseite her mehr Back-up, mehr Instrumentarium, bessere Alternativen“ entwickelt (I 5 13/01). Damit wird die Verbindung zur zweiten Referenzkette im Prozess der konzeptionellen Neuaufstellung der Frankfurter Integrationspolitik deutlich - der Veränderung von Konzepten und Begriffen aus der Migrationsforschung, die für Stadtpolitik mobilisierbar sind.

Wenn im Zitat oben die Fixierung auf „diesen Integrationsbegriff und diese Esser-Definitionen" (I 5 13/o1) problematisiert wird, dann ist damit die Parallele zwischen staatlicher Regulation von und wissenschaftlicher Wissensproduktion zu (Post-)Migration angesprochen. Hartmut Essers handlungstheoretische Variante der Assimilationstheorie hat nicht nur nachhaltig die deutschsprachige Migrationssoziologie geprägt, sein Modell verfügt auch über eine „offensichtliche Deutungshoheit innerhalb der staatlichen bundesrepublikanischen Integrationspolitik“ (Aumüller 2009: 106) - wie im zweiten Abschnitt ausgeführt mit neuem Schwung seit den Reformen des Zuwanderungsrechts. ,Esser ' lässt sich im Zitat dann als Symbol für wissenschaftliche Perspektiven verstehen, die (Post-)Migration in direkter Abhängigkeit zur ontologisierten Form des Nationalstaats konzipieren, denn das wissenschaftliche Integrationsparadigma lässt sich als Quintessenz des ,methodologischen Nationalismus' verstehen (Wimmer/Glick Schiller 2002).

Mit den „völlig anderen Perspektiven“ der Migrationsforschung, die für die Entwicklung des Frankfurter Konzepts eine Rolle spielen sollten, sind also Arbeiten gemeint, die sich aus der Kritik des methodologischen Nationalismus entwickelt haben - postnationale Methodologien zur Beschreibung von (Post-)Migration und Vergesellschaftungsprozessen: allen voran die Transnationalismusforschung, neben Perspektiven wie beispielsweise der Autonomie der Migration, Hybridität, Kosmopolitismus, (Super-)Vielfalt und Transkulturalismus (vgl. Hess/Moser 2009: 13). Als ,postnational' lassen sich diese Perspektiven nicht bezeichnen, weil sie notwendigerweise die Rolle von Nationalstaaten und Nationalismus in der Prägung von transnationaler Migration und Gesellschaften negieren, sondern weil sie diese nicht methodologisch voraussetzen. In diesen Perspektiven wird Migration nicht mehr dichotom auf die als Container begriffene, Herkunftsgesellschaft' oder ,Aufnahmegesellschaft' reduziert, vielmehr wird (Post-)Migration als dauerhafter Bestandteil von Vergesellschaftung unter Globalisierungsbedingungen begriffen (vgl. bspw. Glick Schiller 2007, Geisen 2010).

Dem Dreieck aus, Reformdruck', der spezifischen Tradition der Frankfurter Integrationspolitik als persuasives Programm gegen die Tradition des ,Nichteinwanderungslandes“ sowie der damit verbundenen Bezugnahme auf neue Perspektiven der Migrationsforschung entsprang also der Prozess zur Erarbeitung des Frankfurter Integrationskonzepts. In der Folge wurde der Auftakt mit einem wissenschaftlichen Leitbildentwurf gestaltet, für den keine gesicherten Expert_innen der angewandten Integrationsforschung 
nach Frankfurt geholt wurden. Mit den Kulturanthropolog_innen Regina Römhild und Steven Vertovec als dezidierten Vertreter_innen postnationaler Methodologien und einer Forschung „aus der Perspektive der Migration“ (Römhild 2009) wurde die Expertise stattdessen zu einem Versuch, „Begriffe aus solchen transnationalen Perspektiven für die Stadtpolitik denkbar zu machen" (I 4 12/10).

Die Expertise mobilisierte also postnationale Methodologien für eine Beschreibung der Stadt der Vielfalt (global city, Transnationalismus, Supervielfalt und Milieus der Vielfalt in Frankfurt, Römhild/Vertovec 2009: 30ff.) und artikulierte diese mit bestehenden Diskursen der Integrationspolitik sowie der unternehmerischen Stadt in Bezug auf Frankfurt. Dieser ,postnationale‘ Auftakt des Frankfurter Prozesses zur Erstellung eines Integrationskonzepts war letztlich möglich geworden, weil die Fortschreibung der devianten Frankfurter Tradition der Integrationspolitik und die damit implizierten, in der (deutschen) Migrationsforschung ebenso devianten ,ganz anderen' Perspektiven im Kontext des neoliberalen Reformdrucks auch Distinktionsgewinne versprachen. Das ,Frankfurter Modell hatte der Stadtpolitik schließlich nach dem Abstreifen seiner ,rebellischen“ grünen Anfänge lange Zeit den Platz als Vorreiterin kommunaler Integrationspolitiken sichern können (Aybek/Straßburger 2009). Den State of the Art der Forschung in stadtpolitische Konzepte zu übersetzen, versprach diesen vergangenen Erfolg zu erneuern. Politisch sprach also nichts gegen diesen Versuch - die Dezernentin „war erst mal total begeistert“ (I 5 13/o1). Im weiteren Prozess musste das wissenschaftliche Leitbild an die Erfordernisse des interkommunalen Vergleichs und der politischen Durchsetzung in Dezernaten und Magistrat angepasst werden (I 4 12/10, I 5 13/01). Trotzdem konnten sich die alternativen Referenzketten in den Diskussionsprozessen um das Konzept, im Konzept selbst und vor allem als take home message in den Präsentationen des fertigen Konzepts durch die Mitarbeiter_innen des AmkA und des Dezernats in der Folge artikulieren (I 5 13/o1, FN 13/3-3).

Das Vorwort der Dezernentin zu dem Konzept beginnt mit der Formulierung: „zu Hause zu sein statt integriert“ (Stadt Frankfurt 2011:9; H. d. V.). Die Provokation dieses Satzes als Einleitung eines städtischen Integrationskonzepts erinnert nicht zufällig an kritische Kommentare und Initiativen zum Integrationsparadigma der letzten Jahre: in Frankfurt etwa die „StattIntegration“-Konferenzen, die das Deutsch-Türkische Jugendwerk ausrichtete (Topçu 2011); im weiteren politischen Feld beispielsweise „no integración“ (kanak attak 2002), „Integration? Nein danke!“ (Plattform gegen Rassismus 2010) oder „Demokratie statt Integration“ (kritnet 2010), im wissenschaftlichen Feld zum Beispiel „No integration?!“(Hess/Moser 2009) oder „Vergesellschaftung statt Integration“(Geisen 2010). Das Grundnarrativ des Konzepts - „Vielfalt als Tatsache“ (Stadt Frankfurt 2011: 15) - wird hier zur Voraussetzung jedes Sprechens über Integration (und bedeutet dann eigentlich auch hier: „Diversity statt Integration“, Terkessidis 2008; H. d. V.).

\section{Ausblick}

Die zentrale Referenz auf, Vielfalt als Tatsache“ im Frankfurter Integrationskonzept stellt sich als programmatischer Bruch mit den alten, 
nationalen Mustern zwischen rassistischem Ausschluss und kulturalistischem Integrationsparadigma dar. Dieser Bruch erfolgt im Kontext der neoliberalen Rationalitäten, in denen Stadtpolitik sich heute artikulieren muss: vom interkommunalen Vergleich über das neue Steuerungsmodell bis zum ökonomischen Raster von Stadtpolitik, in dessen Linien sie die Bevölkerung der Stadt im Allgemeinen zu ,aktiven Bürger_innen' zu machen sucht. Citizenship kann sich in kommunalen Integrationskonzepten (wie im Frankfurter Fall) in einer Weise artikulieren, die über ihre neoliberale Genese hinausweist, indem (Post-)Migration als selbstverständlicher Bestandteil einer ,vielfältigen“ Bürgerschaft repräsentiert wird. Mit dieser Repräsentation verändern sich wie gezeigt die urban politics of citizenship - aber das ist nicht gleichzusetzen mit einer Veränderung von citizenship als substanzielle Teilhabe.

Eine Beobachtung von Enis Gülegen, Vorsitzender der Frankfurter Kommunalen Ausländer- und Ausländerinnenvertretung, zeigt dann nicht nur die Möglichkeit einer politischen Referenzierung dieser Politiken im Streit für eine gleichberechtigte Teilhabe in der Stadt, sondern auch die Ambivalenz, die aus dieser Programmatik für solche Kämpfe entsteht:

„Wir lesen im Monitoringbericht zum Beispiel, dass Migranten in Frankfurt für schlechtere Wohnungen höhere Mietzinsen zahlen. [...] Das ist ein Bericht des Magistrats, es ist nicht irgendein Bauchgefühl, was wir als Migranten seit Jahrzehnten sagen - den Eindruck hatten wir ja immer. [...] Wir müssen immer höhere Mieten zahlen und wir bekommen immer die schlechteren Wohnungen. [...] Das Besondere ist aber jetzt, dass der Magistrat das sagt. Und da erwartet man natürlich: Oh, jetzt wird das in der Stadtverwaltung zum Thema gemacht, alle stehen auf und sagen: ,Wir erlauben nicht, dass die Frankfurterinnen und Frankfurter, die vor 30, 40 Jahren hergekommen sind, hier so diskriminiert werden.' Es ist aber nichts passiert.“ (FN 13/10-3)

Nach dem Muster des neuen Steuerungsmodells und seiner ,Produktorientierung' müssten die Ergebnisse des Monitorings in die Steuerung der Verwaltung zurückfließen und folglich müssten die diskriminierenden Strukturen des Wohnungsmarktes in Frankfurt offensiv von der Stadt zum Gegenstand der Intervention gemacht werden. Im Bereich des Arbeitsmarktes oder der Bildung, für die das Monitoring ebenso diskriminierende Strukturen ausweist (Stadt Frankfurt 2012: 64ff. \& 106ff.), gilt das umso mehr. Denn diese Bereiche wären selbst in der engen Fassung einer ökonomischen ,Chancengleichheit‘ als Voraussetzung für die Entfaltung der aktiven Stadtbürger_innen zentral für Antidiskriminierungsarbeit - es ist aber auch hier „nichts passiert“.

Sarah Ahmed hat eine ähnliche Beobachtung für die Implementation von Diversity-Politiken an Hochschulen damit erklärt, dass unter neoliberalen Umständen schon die Erstellung von Konzepten selbst als Erfolg verkauft werden kann: „You end up doing the document rather than doing the doing“ (2011). Weil sich Politiken im Namen von, Vielfalt' dann auf das ,exzellente‘ Management von Dokumenten beschränken, werden rassistische Strukturen in der eigentlichen Verwaltungsarbeit ignoriert, selbst wenn diese in Konzepten und Monitorings offen thematisiert werden. Aber 
daraus ist nicht zu schließen, dass die Repräsentation von Vielfalt oder die Thematisierung von Diskriminierung als ,Sonntagsreden“ abzutun wären. Nicht, weil große Hoffnung besteht, dass die Städte dem Versprechen, das die Repräsentation der Vielfalt beinhaltet, einfach nachkommen. Aber die Abwesenheit entsprechender Interventionen lässt sich durch sie politisch skandalisieren, die Dokumente können „Ärger verursachen“ (ebd.: 135). Analytisch wäre dann zu klären, wo und wie sie das tun (oder nicht), indem man verfolgt, „wie solche Dokumente kursieren, wie sie wandern, wo sie stecken bleiben" (ebd.). In politischer Hinsicht unterstreicht das noch einmal das Anliegen dieses Textes, die urban politics of citizenship aktueller städtischer Integrationspolitiken nicht einfach als neoliberal abzutun. Die Programme entstehen in einem neoliberalen Kontext, aber dies steht ihrem Gebrauch für Kämpfe um urban citizenship als substanzielle Teilhabe nicht entgegen.

\section{Endnoten}

[1] Der Begriff der (Post-)Migration dient hier der Reflexion der reichlich ungenauen (staatlichen) Problematisierung eines Teils der in Deutschland lebenden Menschen, die mit Bezug auf ihre (teilweise Generationen zurückliegende) Migrationsgeschichte als (defizitäre) Abweichung von der national normierten Gesellschaft konstruiert werden. Er verweist dabei auf alternative Entwürfe einer, postmigrantischen' Gesellschaft (bspw. Langhoff 2011, Terkessidis 2013, Yildiz 2010). Alte, neue und andauernde Migrationen sind heute alltägliche Realität und prägende Kraft von Vergesellschaftung. Das zeigt sich besonders in Städten wie Frankfurt, wo 67 Prozent der unter Sechsjährigen einen sogenannten Migrationshinweis haben. Die auch mit diesem Begriff häufig nach wie vor verbundene Konnotation der (nationalen) Devianz wirkt dann schlicht absurd - und gerät ins Wanken. Der Begriff der (Post-)Migration markiert hier also auch die These, dass die „Turbulenzen im nationalen Behälter“ (Terkessidis 2013) - die Herausforderung, die die Bewegung der Migration und der transnationale Alltag laufend für das tradierte Selbstverständnis der deutschen Gesellschaft als ,ethnisches Staatsvolk‘ darstellen - sich mittlerweile auch in staatlichen Programmen niederschlagen.

[2] Kelek vertritt eine kulturalistische Islamkritik, aus der sie ableitet, dass der Staat ,Integration“ als Forderung der Anpassung an die Mehrheitsgesellschaft durchsetzen müsse (Rommelspacher 2010). Thilo Sarrazins „Deutschland schafft sich ab“ begrüßte sie dann auch als „Befreiungsschlag“ (Friedrich 2011: 12).

[3] Gegen die polemische Überzeichnung der Kritik Keleks argumentiert jedenfalls die Integrationsdezernentin (Eskandari-Grünberg 2011).

[4] Die Argumentation hier wäre ohne die von Sabine Hess und Henrik Lebuhn im März 2013 veranstaltete Konferenz „Stadt und Migration“ nicht zustande gekommen. Auch die Diskussionen im Kontext des Frankfurter Verbundprojekts „Neuordnungen des Städtischen im neoliberalen Zeitalter“ (insbesondere mit Iris Dzudzek), im Göttinger Labor kritische Migrations- und Grenzregimeforschung sowie die Hinweise und äußerst konstruktive Kritik der beiden anonymen Gutachter_innen des Textes haben viel zur hier formulierten Argumentation beigetragen. Ihnen und der Redaktion von $\mathrm{s} u \mathrm{~b} \backslash \mathrm{u} \mathrm{r}$ b a $\mathrm{n}$ möchte ich danken. Gleiches gilt für die Unterstützung durch Mitarbeiter_innen des Frankfurter Amts für multikulturelle Angelegenheiten und deren Offenheit im Rahmen der Feldforschung des zugrunde liegenden Projekts.

[5] Siehe www.neuordnungen.info/migrationspolitik (letzter Zugriff am 22.10.2014).

[6] Während die Einbürgerung von sogenannten Statusdeutschen - also Migrant_innen, die ihre deutsche „Volkszugehörigkeit“ durch „Merkmale wie Abstammung, Sprache, Erziehung, Kultur“ nachweisen können (§6(1) BVFG) - vorgesehen ist, war die Einbürgerung von anderen Migrant_innen bis zum Abschluss der Reform des Staatsangehörigkeitsrechts 1999 praktisch nicht vorgesehen. Damit waren Generationen von im Land lebenden Menschen dauerhaft von der politischen Teilhabe ausgeschlossen 
(bspw. lag die Einbürgerungsquote in den 1980er Jahren bei o,3 Prozent der ausländischen Bevölkerung; Green 2006: 116).

[7] Gemeint sind hier die assimilationstheoretischen Arbeiten des Soziologen Hartmut Esser (s. u.).

\section{Autor_innen}

Der Autor hat an Schnittpunkten von politischer Theorie, Staats- und Stadtforschung zu (Post-)Migration, Rassismus und Neonazismus gearbeitet.

rodatz@geo.uni-frankfurt.de

\section{Literatur}

Ahmed, Sarah (2011): „You end up doing the document rather than doing the doing“. Diversity, Race Equality und Dokumentationspolitiken. In: María do Mar Castro Varela / Nikita Dhawan (Hg.): Soziale (Un)Gerechtigkeit. Kritische Perspektiven auf Diversity, Intersektionalität und Antidiskriminierung. Berlin: Lit-Verlag, 118-137.

Aretz, Hans-Jürgen (2004): Ökonomischer Liberalismus, postmodernes Diversity Management in Unternehmen und der Geist des Kapitalismus. In: Österreichische Zeitschrift für Soziologie 29/3, 3-23.

Attia, Iman (2007): Kulturrassismus und Gesellschaftskritik. In: dies. (Hg.): Orientund Islambilder. Interdisziplinäre Beiträge zu Orientalismus und antimuslimischem Rassismus. Münster: Unrast-Verlag, 5-30.

Aumüller, Jutta (2009): Assimilation. Kontroversen um ein migrationspolitisches Konzept. Bielefeld: transcript.

Aybek, Can M. / Straßburger, Gaby (2009): ,Politik des friedlichen Zusammenlebens‘. Integrationsansatz mit Modellcharakter in Frankfurt/Main. In: Frank Gesemann / Roland Roth (Hg.): Lokale Integrationspolitik in der Einwanderungsgesellschaft. Migration und Integration als Herausforderung von Kommunen. Wiesbaden: VS, 351-365.

Balibar, Étienne (2001): Kommunismus und (Staats-)Bürgerschaft. Überlegungen zur emazipatorischen Politik. http://www.niatu.net/nospoon/texte/balibar_staatsbuergerschaft.pdf (letzter Zugriff am 23.10.2014).

Bauböck, Rainer (2003): Reinventing urban citizenship. In: Citizenship Studies 7/2, 139-160.

Bommes, Michael (2008): „Integration findet vor Ort statt“. Über die Neugestaltung kommunaler Integrationspolitik. In: ders. / Marianne Krüger-Potratz (Hg.): Migrationsreport 2008. Fakten - Analysen - Perspektiven. Frankfurt a. M.: Campus, 159-194.

Brenner, Neil (2003): ,Glocalization' as a state spatial strategy. Urban entrepreneurialism and the new politics of uneven development in western Europe. In: Jamie Peck / Henry Wai-Chung Yeung (Hg.): Remaking the Global Economy. Economic-Geographical Perspectives. London/Thousand Oaks: Sage, 197-215.

Bröckling, Ulrich / Krasmann, Susanne / Lemke, Thomas (2000): Gouvernementalität, Neoliberalismus und Selbsttechnologien. In: dies. (Hg.): Gouvernementalität der Gegenwart. Studien zur Ökonomisierung des Sozialen. Frankfurt a. M.: Suhrkamp, 7-40.

Clarke, Nick (2012): Urban policy mobility, anti-politics, and histories of the transnational municipal movement. In: Progress in Human Geography 36/1, 25-43.

Dohse, Knuth (1981): Ausländische Arbeiter und bürgerlicher Staat. Genese und Funktion von staatlicher Ausländerpolitik und Ausländerrecht - vom Kaiserreich bis zur Bundesrepublik Deutschland. Königstein: Hain.

Dölemeyer, Anne / Rodatz, Mathias (2010): Diskurse und die Welt der Ameisen. Foucault mit Latour lesen (und umgekehrt). In: Robert Feustel / Maximilian Schochow (Hg.): Zwischen Sprachspiel und Methode. Perspektiven der Diskursanalyse. Bielefeld: transcript, 197-220.

Eskandari-Grünberg, Nargess (2011): Die Verantwortung des Einzelnen. In: Frankfurter Allgemeine Zeitung, 24.1.2011.

Ferguson, James (2009): The uses of neoliberalism. In: Antipode 41/S1, 166-184.

Foucault, Michel (2001): In Verteidigung der Gesellschaft. Vorlesungen am Collège de France (1975-76). Frankfurt a.M: Suhrkamp. 
Friedrich, Sebastian (2011): Rassismus in der Leistungsgesellschaft. In: ders. (Hg.): Rassismus in der Leistungsgesellschaft. Analysen und kritische Perspektiven zu den rassistischen Normalisierungsprozessen in der „Sarrazindebatte“. Münster: edition assemblage, 8-38.

Ganser, Karl (1974): Regionalisierte Ausländerpolitik. Einführung. In: Informationen zur Raumentwicklung 1974/2, 33-36.

Garcia, Soledad (1996): Cities and citizenship. In: International Journal of Urban and Regional Research 20/1, 7-21.

Geisen, Thomas (2010): Vergesellschaftung statt Integration. Zur Kritik des IntegrationsParadigmas. In: Paul Mecheril / İnci Dirim / Mechtild Gomolla / Sabine Hornberg / Krassimir Stijanov (Hg.): Spannungsverhältnisse. Assimilationsdiskurse und interkulturell-pädagogische Forschung. Münster: Waxmann, 13-34.

Gesemann, Frank / Roth, Roland / Aumüller, Jutta (2012): Stand der kommunalen Integrationspolitik in Deutschland. Studie erstellt für das Bundesministerium für Verkehr, Bau und Stadtentwicklung und die Beauftragte der Bundesregierung für Migration, Flüchtlinge und Integration. Berlin: Bundesministerium für Verkehr, Bau und Stadtentwicklung/Die Beauftragte der Bundesregierung für Migration, Flüchtlinge und Integration.

Glick Schiller, Nina (2007): Beyond the nation-state and its units of analysis: Towards a new research agenda for migration studies. Essentials of migration theory. COMCAD Working Paper 33/2007. Bielefeld: Center on Migration, Citizenship and Development (COMCAD).

Green, Simon (2006): Zwischen Kontinuität und Wandel. Migrations- und Staatsangehörigkeitspolitik. In: Manfred Schmidt / Reimut Zohlnhöfer (Hg.): Regieren in der Bundesrepublik Deutschland. Wiesbaden: VS, 113-134.

Gutiérrez Rodríguez, Encarnación (2003): Gouvernementalität und die Ethnisierung des Sozialen. In: Marianne Pieper / dies. (Hg.): Gouvernementalität. Ein sozialwissenschaftliches Konzept in Anschluss an Foucault. Frankfurt a. M.: Campus, 161-178.

Harvey, David (1989): From managerialism to entrepreneurialism. The transformation in urban governance in late capitalism. In: Geografiska Annaler, Series B, Human Geography 71/1, 3-17.

Häußermann, Hartmut / Kapphan, Andreas (2008): Integrationspolitik der Städte - ein Paradigmenwechsel. In: Michael Bommes / Marianne Krüger-Potratz / Rat für Migration (Hg.): Migrationsreport 2008. Frankfurt a. M.: Campus, 15-28.

Hentges, Gudrun (2002): Das Plädoyer für eine „deutsche Leitkultur“ - Steilvorlage für die extreme Rechte? In: Christoph Butterwegge (Hg.): Themen der Rechten - Themen der Mitte. Zuwanderung, demografischer Wandel und Nationalbewusstsein. Opladen: Leske + Budrich, 95-121.

Hess, Sabine / Moser, Johannes (2009): Jenseits der Integration. Kulturwissenschaftliche Betrachtungen einer Debatte. In: Sabine Hess / Jana Binder / Johannes Moser (Hg.): No integration?! Kulturwissenschaftliche Beiträge zur Integrationsdebatte in Europa. Bielefeld: transcript, 11-26.

Holston, James / Appadurai, Arjun (1996): Cities and citizenship. In: Public Culture 8/2, 187-204.

Isin, Engin F. / Siemiatycki, Myer (1999): Fate and faith. Claiming urban citizenship in immigrant Toronto. Working Paper No. 8. Toronto: Joint Centre of Excellence for Research on Immigration and Settlement.

kanak attak (2002): no integración. Recht auf Legalisierung. Globalisierungskritik und Antirassismus. http://www.kanak-attak.de/ka/down/pdf/kanak-attak_2002.pdf (letzter Zugriff am 2.10.2010).

Karakayali, Serhat / Tsianos, Vassilis (2002): Migrationsregimes in der Bundesrepublik Deutschland. Zum Verhältnis von Staatlichkeit und Rassismus. In: Alex Demirovic / Manuela Bojadzijev (Hg.): Konjunkturen des Rassismus. Münster: Westfälisches Dampfboot, 246-267.

Kelek, Necla (2011): „Das ist kein Integrationskonzept“. In: Frankfurter Allgemeine Zeitung, 11.1.2011.

Kessl, Fabian / Krasmann, Susanne (2005): Sozialpolitische Programmierungen. In: Fabian Kessl / Christian Reutlinger / Susanne Maurer / Oliver Frey (Hg.): Handbuch Sozialraum. Wiesbaden: VS, 227-246.

kritnet - Netzwerk kritische Migrations- und Grenzregimeforschung (2010): Demokratie statt Integration. http://demokratie-statt-integration.kritnet.org (letzter Zugriff am 1.10.2010). 
Langhoff, Schermin (2011): Die Herkunft spielt keine Rolle. „Postmigrantisches“ Theater im Ballhaus Naunynstraße. http://www.bpb.de/gesellschaft/kultur/kulturellebildung/60135/interview-mit-shermin-langhoff?p=all (letzter Zugriff am 22.3.2012).

Lanz, Stephan (2009): In unternehmerische Subjekte investieren. Integrationskonzepte im Workfare-Staat. Das Beispiel Berlin. In: Sabine Hess / Jana Binder / Johannes Moser (Hg.): No integration?! Kulturwissenschaftliche Beiträge zur Integrationsdebatte in Europa. Bielefeld: transcript, 105-122.

Lebuhn, Henrik (2010): Das Neue Steuerungsmodell und die (Markt-)Logik städtischer Verwaltungen. In: Z - Zeitschrift Marxistische Erneuerung 21/83, 35-45.

Lebuhn, Henrik (2013): Migration - Recht - Citizenship. Potentiale und Grenzen eines kritischen Diskurses. In: Paul Mecheril / Oscar Thomas-Olalde / Claus Melter / Susanne Arens / Elisabeth Romaner (Hg.): Migrationsforschung als Kritik? Konturen einer Forschungsperspektive. Wiesbaden: VS, 231-244.

Leggewie, Claus (2011): Das Amt für Multikulturelle Angelegenheiten. Ein persuasives Programm. In: Claus Leggewie (Hg.): Multikulti. Spielregeln für die Vielvölkerrepublik. Salzhemmendorf: Blumenkamp, 71-85.

Meier-Braun, Karl-Heinz (2013): Einleitung: Deutschland Einwanderungsland. In: KarlHeinz Meier-Braun / Reinhold Weber (Hg.): Deutschland Einwanderungsland. Begriffe - Fakten - Kontroversen. Stuttgart: Kohlhammer, 15-27.

Mestre Vives, Laura (1998): Wer, wie, über wen? Eine Untersuchung über das Amt für multikulturelle Angelegenheiten. Frankfurt a.M: Centaurus.

Mick, Günter (1989): Hauff hat Frankfurter beleidigt. In: Frankfurter Allgemeine Zeitung, 2.3.1989.

Münch, Sybille (2010): Integration durch Wohnungspolitik? Zum Umgang mit ethnischer Segregation im europäischen Vergleich. Wiesbaden: VS.

Nyers, Peter (2010): No one is illegal between city and nation. In: Studies in Social Justice 4/2, 127-143.

Osborne, Thomas / Rose, Nikolas (1999): Governing cities. Notes on the spatialisation of virtue. In: Environment and Planning D: Society and Space 17/6, 737-760.

Peck, Jamie (2001): Neoliberalizing states. Thin policies/hard outcomes. In: Progress in Human Geography 25/3, 445-455.

Peck, Jamie / Theodore, Nik (2010): Mobilizing policy. Models, methods, and mutations. In: Geoforum 41/2, 169-174.

Plattform gegen Rassismus (2010): Integration? Nein danke! http://integrationneindanke. files.wordpress.com/2010/o4/integration_nein_danke_deutsch.pdf (letzter Zugriff am 2.10.2012).

Pütz, Robert / Rodatz, Mathias (2013): Kommunale Integrations- und Vielfaltskonzepte im Neoliberalismus. Zur strategischen Steuerung von Integration in deutschen Großstädten. In: Geographische Zeitschrift 101/3+4, 166-183.

Reimer, Kathrin (2013): Rechte Ideologie und soziale Frage. Soziale Arbeit und Politische Bildung in Zeiten des rechtspopulistischen Neoliberalismus. In: Friedrich Burschel / Uwe Schubert / Gerd Wiegel (Hg.): Der Sommer ist vorbei... Vom „Aufstand der Anständigen“ zur „Extremismus-Klausel“. Beiträge zu 13 Jahren „Bundesprogramme gegen Rechts“. Münster: edition assemblage, 29-50.

Rodatz, Mathias (2012): Produktive „Parallelgesellschaften“. Migration und Ordnung in der (neoliberalen) „Stadt der Vielfalt“. In: Behemoth 5/1, 70-103.

Rommelspacher, Birgit (2010): Islamkritik und antimuslimische Positionen am Beispiel von Necla Kelek und Seyran Ateş. In: Thorsten G. Schneiders (Hg.): Islamfeindlichkeit. Wiesbaden: VS, 447-469.

Ronneberger, Klaus / Tsianos, Vassilis (2009): Panische Räume. Das Ghetto und die „Parallelgesellschaft“. In: Sabine Hess / Jana Binder / Johannes Moser (Hg.): No integration?! Kulturwissenschaftliche Beiträge zur Integrationsdebatte in Europa. Bielefeld: transcript, 137-152.

Rose, Nikolas (2000): Governing cities, governing citizens. In: Engin F. Isin (Hg.): Democracy, Citizenship and the Global City. London: Routledge, 96-109.

Rosol, Marit (2013): Regieren (in) der neoliberalen Stadt. Foucaults Analyse des Neoliberalismus als Beitrag zur Stadtforschung. In: Geographische Zeitschrift 101/3+4, 132-147.

Römhild, Regina (2009): Aus der Perspektive der Migration. Die Kosmopolitisierung Europas. In: Sabine Hess / Jana Binder / Johannes Moser (Hg.): No integration?! Kulturwissenschaftliche Beiträge zur Integrationsdebatte in Europa. Bielefeld: transcript, 207-223. 
Römhild, Regina / Vertovec, Steven (2009): „Frankfurt vernetzt“. Vernetzungs- und Vielfaltspolitik in Frankfurt am Main. In: Stadt Frankfurt (Hg.): Entwurf eines Integrations- und Diversitätskonzepts für die Stadt Frankfurt am Main. Ein Arbeitspapier des Dezernats für Integration. Frankfurt a. M.: Stadt Frankfurt, 20-79.

Sassen, Saskia (1991): The Global City. New York, London, Tokyo. Princeton: Princeton University Press.

Silomon-Pflug, Felix / Heeg, Susanne (2013): Neoliberale Neuordnung städtischer Verwaltungen am Beispiel des Liegenschaftsfonds Berlin. In: Geographische Zeitschrift 101/3+4, 184-200.

Schipper, Sebastian (2013): Genealogie und Gegenwart der unternehmerischen Stadt. Neoliberales Regieren in Frankfurt am Main, 1960-2010. Münster: Westfälisches Dampfboot.

Stadt Berlin (2007): Vielfalt fördern - Zusammenhalt stärken. Das Berliner Integrationskonzept: Handlungsfelder, Ziele, Leitprojekte. http://www.berlin.de/imperia/md/ content/lb-integration-migration/publikationen/berichte/integrationskonzept_2007_ bf.pdf?start\&ts=1301067806\&file=integrationskonzept_2007_bf.pdf (letzter Zugriff am 23.10.2014).

Stadt Frankfurt (2011): Vielfalt bewegt Frankfurt. Integrations- und Diversitätskonzept für Stadt, Politik und Verwaltung. http://www.frankfurt.de/sixcms/media.php/738/Integrationskonzept_2011.pdf (letzter Zugriff am 23.10.2014).

Stadt Frankfurt (2012): Frankfurter Integrations- und Diversitätsmonitoring 2012. Frankfurt a. M: Magistrat der Stadt Frankfurt a. M.

Stadt Leipzig (2006): Die Integration der Migranten in Leipzig als Querschnittsaufgabe. Bericht des Referats Ausländerbeauftragter. Stadt Leipzig: Dezernat Allgemeine Verwaltung/Referat Ausländerbeauftragter.

Terkessidis, Mark (2008): Diversity statt Integration. Kultur- und integrationspolitische Entwicklungen der letzten Jahre. In: Kulturpolitische Mitteilungen IV/2008/123, 47-52.

Terkessidis, Mark (2013): Nationale Turbulenzen. Unromantische Betrachtungen über postmigrantische Urbanität und Kunstproduktion. http://www.deutscher-pavillon. org/2013/mark-terkessidis-nationale-turbulenzen-unromantische-betrachtungen-uberpostmigrantische-urbanitat-und-kunstproduktion-2/ (letzter Zugriff am 16.12.2013).

Topçu, Canan (2007): Wie eine Gebetsmühle. http://www.fr-online.de/spezials/wie-einegebetsmuehle,1472874,2739068.html (letzter Zugriff am 20.2.2013).

Topçu, Canan (2011): „In Diskussionen gibt es viel Verachtung und Überheblichkeit“. Interview zur Integrationsdebatte in Frankfurt. http://www.fr-online.de/frankfurt/ interview-zur-integrationsdebatte-in-frankfurt--in-diskussionen-gibt-es-vielverachtung-und-ueberheblichkeit-,1472798,5061922.html (letzter Zugriff am 2.3.2011).

Tsianos, Vassilis (2013): Urbane Paniken. Zur Entstehung des antimuslimischen Urbanismus. In: Duygu Gürsel / Zülfukar Çetin / Allmende e.V. (Hg.): Wer Macht Demo_ kratie? Kritische Beiträge zu Migration und Machtverhältnissen. Münster: edition assemblage, 23-43.

Welz, Gisela (1998): Wie verwaltet man die kulturelle Vielfalt einer Weltstadt? Das Frankfurter Amt für multikulturelle Angelegenheiten. In: Ina-Maria Greverus / Johannes Moser / Heinz Schilling / dies. (Hg.): Frankfurt am Main. Ein kulturanthropologischer Stadtführer. Frankfurt a. M.: Institut für Kulturanthropologie und Europäische Ethnologie der Universität Frankfurt, 33-48.

Wimmer, Andreas / Glick Schiller, Nina (2002): Methodological nationalism and beyond. Nation-state building, migration and the social sciences. In: Global Networks 2/4, 301-334.

Yildiz, Erol (2010): Die Öffnung der Orte zur Welt und postmigrantische Lebensentwürfe. In: SWS-Rundschau 2010/3, 318-339.

\section{„Migration is a fact in this city“: Urban politics of citizenship in the neoliberal city}

The paper discusses the politics of citizenship articulated in recent city strategies of integration policy. As the German paradigm of 'integration' is being adopted by the 'entrepreneurial city', the tradition of national normalization in urban policy loses its function. Corresponding city strategies 
problematize (post-)migrants as responsible for their self-integration and the development of their communities for the benefit of the city. The paper suggests to consider the neoliberal context of these urban politics of citizenship not just as a projection for negative critique, but in its potential use for substantial gains in urban citizenship. A closer look at the concept of the city of Frankfurt reveals that it mobilizes post-national epistemologies from the social sciences and develops a representation of the city's 'diversity' that may well be used to present political demands. 
